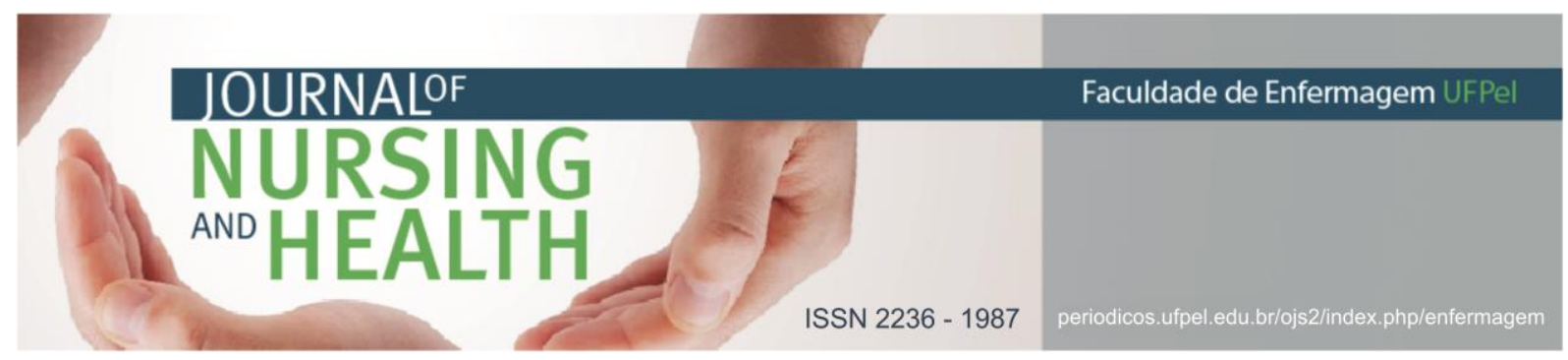

ARTIGO DE REVISÃO

\title{
Realização da evolução de enfermagem em âmbito hospitalar: uma revisão sistemática
}

\author{
Carrying out the evolution of nursing in hospitals: a systematic review
}

Realización de la evolución de la enfermería en hospitales: una revisión sistemática

Lima, Oséias José Lopes'; Lima, Ângela Roberta Alves²

\begin{abstract}
RESUMO
Objetivo: realizar uma revisão sistemática de literatura sobre a frequência da realização da evolução de enfermagem nas unidades de internação hospitalar e a adequação às prerrogativas legais. Métodos: revisão sistemática, com coleta de dados na biblioteca eletrônica Scientific Eletronic Library Online SCiELO, com os descritores controlados: registros de enfermagem; cuidados de enfermagem, processos de enfermagem e o descritor não controlado: evolução. As buscas ocorreram no mês de julho, 2016. Resultados: evidenciaram que produção sobre evolução de enfermagem nos periódicos pesquisados, é pouco expressiva. Considerações Finais: a Sistematização da Assistência de Enfermagem, método de consenso entre os enfermeiros, amplamente conhecido e legalmente instituído, ainda enfrenta uma série de inconsistências e irregularidades. Identificou-se um grande volume de inconformidades na implementação e execução dessa sistemática, revelando um oportuno espaço de atuação para a auditoria de enfermagem a qual poderia contribuir para o exercício da enfermagem de forma científica, segura, legal e de qualidade.
\end{abstract}

Descritores: Cuidados de enfermagem; Registros de enfermagem; Processo enfermagem.

\begin{abstract}
Objective: to conduct a systematic review of the literature on the frequency of the evolution of nursing in hospital admission units and the adequacy to legal prerogatives. Methods: systematic review with data collection based on Scientific Electronic Library Online Scielo, with the controlled descriptors: nursing care, nursing process and nursing records, and the uncontrolled descriptor: evolution. The searches took place in July 2016. Results: evidenced that production on nursing evolution in the journals surveyed, is not very expressive. Final considerations: Systematization of Nursing Assistance, method of consensus among nurses, widely known and legally established, still faces a number of inconsistencies and irregularities in its implementation and enforcement. The existence of a large volume of non-compliance in the implementation and execution of this system opens a timely performance space for the audit of nursing which could significantly contribute to the practice of nursing science, safe, legal and quality.

Descriptors: Nursing care; Nursing records; Nursing process.
\end{abstract}

\footnotetext{
${ }^{1}$ Enfermeiro. Especialista em Saúde da Família e Auditoria em Enfermagem. Enfermeiro da Universidade Federal de Rio Grande. Rio Grande, Rio Grande do Sul, Brasil. Email: oseiaseangela@hotmail.com http://orcid.org/00000001-6762-7498

2 Enfermeira. Mestre em Ciências da Saúde. Doutoranda em Enfermagem pela Universidade Federal de Pelotas. Enfermeira, Prefeitura Municipal de Pelotas, Pelotas, Rio Grande do Sul, Brasil. Email: angelarobertalima@hotmail.com http: //orcid.org/0000-0003-1328-5570
} 


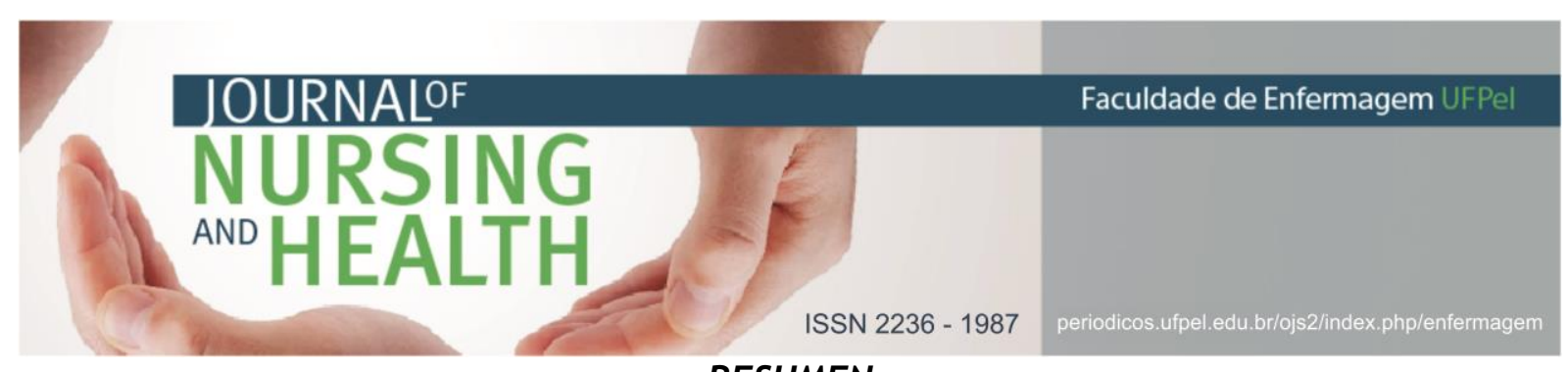

\section{RESUMEN}

Objetivo: realizar revisión sistemática sobre la frecuencia de la evolución de la enfermería en unidades hospitalarias y el cumplimiento de las prerrogativas legales Métodos: revisión sistemática con la compilación de datos de la biblioteca de Scientific Electronic Library Online Scielo, con los descriptores controlados: cuidados de enfermería, proceso de enfermería y registros de enfermería y lo descriptor no controlado: evolución. Las búsquedas se ocurrieran en julio de 2016. Resultados: Evidenciaron que la producción sobre evolución de enfermería en los periódicos, es poco expresiva Consideraciones Finales: Sistematización de la Asistencia de Enfermería, método de consenso entre las enfermeras, conocidas y legalmente instituido, enfrenta una serie de inconsistencias $e$ irregularidades. La existencia de un gran volumen de incumplimiento en la implementación y ejecución de este sistema abre espacio de actuación oportuna para el control de enfermería, que podría contribuir significativamente a la práctica de la enfermería, segura, legal y de calidad.

Descriptores: Atención de enfermería; Registros de enfermería; Procesos de enfermería.

\section{INTRODUÇÃO}

A evolução de enfermagem é o relato diário ou periódico das mudanças que ocorrem com o cliente. ${ }^{1}$ Compõe uma das fases do processo de enfermagem e, mais recentemente, da Sistematização da Assistência de Enfermagem (SAE), proporcionando o registro das ações profissionais, do estado de saúde e de todas as mudanças ocorridas com o cliente.

O registro, em prontuário único, da assistência prestada, abrange aspectos referentes à evolução clínica, procedimentos e cuidados de enfermagem. Esse registro qualifica $o$ cuidado, respalda ética e legalmente o profissional, o cliente e a instituição. Serve como fonte de investigação, instrumento de educação, de auditoria e documento legal, além de constituir um dos direitos do cidadão garantido pela Carta dos Direitos dos Usuários de Saúde. ${ }^{2}$

Em 2009, o Conselho Federal de Enfermagem (COFEN) ${ }^{3}$, instituiu mediante a resolução $n^{\circ} 358$, a SAE e a implementação do processo de enfermagem, pautados por um processo técnico, organizado, deliberado e sistemático, desenvolvido em todos os ambientes, públicos ou privados em que ocorre o cuidado profissional de enfermagem.

A evolução ocupa um papel importante na SAE, devido as suas implicações técnicas, éticas e legais que justificam a busca mais apurada pelo aprofundamento do conhecimento acerca da sua natureza e o vislumbre de um panorama que retrate a realidade assistencial hospitalar brasileira.

Destarte a SAE permite a construção dinâmica do espaço profissional, incorpora um sistema próprio de valor social e cultural, corroborando no enfrentamento da inércia, da fragmentação e burocratização do fazer do enfermeiro, incentivando a criatividade, a percepção da realidade, o desenvolvimento do pensamento crítico e a inovação. Contribui para que a organização do cuidado se torne uma prática reflexiva, habilitando os profissionais ao desenvolvimento do raciocínio crítico sobre suas ações. $\mathrm{E}$, evidencia as contribuições da assistência de enfermagem no cuidado à saúde, concorrendo para a produção 


\section{JOURNALOF \\ NURSING \\ ANO HEALTH}

ISSN 2236 - 1987

pergunta da revisão, os critérios de inclusão e exclusão e estratégias de busca dos artigos. Num segundo momento definiu-se a pergunta a qual serviu como guia para a revisão: a evolução de enfermagem é realizada sistematicamente nas unidades de internação hospitalar no Brasil?

A biblioteca utilizada para o rastreamento dos artigos foi a Scientific Eletronic Library Online (SCiELO). Os descritores utilizados foram previamente consultados no Descritores em Ciências da Saúde (DeSC), tendo sido utilizado o descritor controlado: registros de enfermagem e o não controlado: evolução e o booleano "and" e os descritores controlados: Cuidados de Enfermagem; Processos de Enfermagem; Registros de Enfermagem, conforme Quadro 1.

A busca foi realizada no mês de julho de 2016. Encontrou-se 52 estudos, os critérios de inclusão utilizados foram: artigos realizados por enfermeiros, sobre evolução de enfermagem e registros de enfermagem. Em decorrência do interesse pelo conhecimento da produção científica na temática de escolha a busca não foi delimitada quanto ao ano de publicação e nem quanto à metodologia utilizada.

Os critérios de exclusão foram: estudos que não se aplicam em humanos; não realizados pela enfermagem brasileira; que não tratarem de evolução ou registro de enfermagem. Foram somente avaliados artigos realizados pela enfermagem brasileira devido à legislação especifica que a SAE possui no país. 


\section{NURSING \\ ANO HEALTH}

Artigos identificados na Scientific Eletronic Library Online SCiELO

$$
(n=52)
$$<smiles>c1ccccc1</smiles>

Artigos após eliminação dos duplicados $(\mathrm{n}=48)$

Artigos excluídos por não serem brasileiros $(\mathrm{n}=10)$
Artigos excluídos por não tratarem do tema evolução e/ou registro de enfermagem $(n=27)$ $(n=38)$

Artigos completos avaliados para elegibilidade $(n=11)$ $(n=10)$
Artigo excluído por não tratar do tema

$(n=1)$

Quadro 1 - Fluxograma da seleção das teses para a revisão integrativa, baseado no Modelo PRISMA

Após a eliminação de 04 artigos duplicados, restaram 48. Destes 10 foram excluídos por não se tratarem de artigos realizados no território brasileiro, 4 eram colombianos, 2 chilenos, 2 espanhóis e 2 portugueses. Após a análise dos títulos e resumos 27 artigos foram excluídos por não tratarem do tema.

Posteriormente realizou-se a leitura na integra dos artigos, a identificação e a categorização dos estudos9-19 com o intuito de conhecer o delineamento histórico e metodológico. Para isso foi construído um quadro contendo: ano de publicação, tipo de estudo, amostra e resultados encontrados.

Após essa etapa, um artigo de 201218 foi eliminado da análise, devido ao fato de que seu objetivo foi identificar as ações de enfermagem prescritas por enfermeiros nos prontuários de clientes internados, não se relacionando ao tema evolução de enfermagem, portanto a amostra constituiu-se de 10 artigos. 


\section{NURSING \\ AND \\ HEALTH}

ISSN $2236-1987$

\section{RESULTADOS}

A amostra da revisão constituiuse de dez artigos ${ }^{9-17,19}$ publicados, desde 2002 até 2016. A distribuição por ano de publicação ficou assim constituída: Em $2002^{14}$, $2005^{10} 2006^{13}$, $2008^{17}, 2009^{12}, 2010^{16}, 2012^{9,15} 2015^{19} \mathrm{e}$ $2016^{11}$, conforme Quadro 2.

\begin{tabular}{|c|c|c|c|}
\hline Artigo & Ano & Tipo de Estudo & Revista \\
\hline $\begin{array}{l}\text { Implementação do processo de enfermagem } \\
\text { em uma unidade de radioterapia: elaboração } \\
\text { de instrumento para registro. }\end{array}$ & 2002 & $\begin{array}{l}\text { Relato } \\
\text { experiência }\end{array}$ & $\begin{array}{l}\text { Revista Latino Americana de } \\
\text { Enfermagem }\end{array}$ \\
\hline $\begin{array}{l}\text { Avaliação da realização e do registro da } \\
\text { Sistematização da Assistência de Enfermagem } \\
\text { (SAE) em um hospital universitário. }\end{array}$ & 2005 & $\begin{array}{l}\text { Estudo descritivo, } \\
\text { de caráter } \\
\text { retrospectivo }\end{array}$ & $\begin{array}{l}\text { Revista Brasileira } \\
\text { Enfermagem }\end{array}$ \\
\hline $\begin{array}{l}\text { Sistematização da assistência de enfermagem } \\
\text { em unidade de reabilitação segundo o modelo } \\
\text { conceitual de horta. }\end{array}$ & 2006 & $\begin{array}{l}\text { Relato } \\
\text { experiência }\end{array}$ & $\begin{array}{l}\text { Revista Brasileira } \\
\text { Enfermagem }\end{array}$ \\
\hline $\begin{array}{l}\text { Anotações de enfermagem em uma unidade } \\
\text { cirúrgica de um hospital escola }\end{array}$ & 2008 & $\begin{array}{l}\text { Estudo inter- } \\
\text { relacional, } \\
\text { retrospectivo }\end{array}$ & $\begin{array}{l}\text { Revista Brasileira } \\
\text { Enfermagem }\end{array}$ \\
\hline $\begin{array}{l}\text { Avaliação da qualidade dos registros de } \\
\text { enfermagem no prontuário por meio da } \\
\text { auditoria. }\end{array}$ & 2009 & $\begin{array}{l}\text { Estudo descritivo, } \\
\text { retrospectivo }\end{array}$ & $\begin{array}{l}\text { Revista Acta Paulista de } \\
\text { Enfermagem }\end{array}$ \\
\hline $\begin{array}{l}\text { Análise da implementação da Sistematização } \\
\text { da Assistência de Enfermagem em uma } \\
\text { unidade de reabilitação. }\end{array}$ & 2010 & $\begin{array}{l}\text { Estudo } \\
\text { abordagem } \\
\text { quantitativa, } \\
\text { exploratória } \\
\text { retrospectiva } \\
\end{array}$ & $\begin{array}{l}\text { Revista Brasileira } \\
\text { Enfermagem }\end{array}$ \\
\hline $\begin{array}{l}\text { Avaliação dos registros de enfermeiros em } \\
\text { prontuários de pacientes internados em } \\
\text { unidade de clínica médica. }\end{array}$ & 2012 & $\begin{array}{l}\text { Não apresenta o } \\
\text { tipo de estudo }\end{array}$ & $\begin{array}{l}\text { Revista Acta Paulista de } \\
\text { Enfermagem }\end{array}$ \\
\hline $\begin{array}{l}\text { Tempo despendido na execução do processo de } \\
\text { enfermagem em um centro de tratamento } \\
\text { intensivo. }\end{array}$ & 2012 & $\begin{array}{l}\text { Descritivo, } \\
\text { observacional } \\
\text { exploratório }\end{array}$ & Revista da Escola Anna Nery \\
\hline $\begin{array}{l}\text { Manual de monitoreo de la calidad de los } \\
\text { registros de enfermería en la atención } \\
\text { domiciliaria. }\end{array}$ & 2015 & Estudo de validação & $\begin{array}{l}\text { Revista Brasileira } \\
\text { Enfermagem }\end{array}$ \\
\hline $\begin{array}{l}\text { Termo } \\
\text { de ev }\end{array}$ & 2016 & $\begin{array}{l}\text { Estudo } \\
\text { quantitativo, } \\
\text { descritivo }\end{array}$ & $\begin{array}{l}\text { Revista Brasileira } \\
\text { Enfermagem }\end{array}$ \\
\hline
\end{tabular}

Quadro 2 - Apresentação dos artigos que compõem a amostra

No que refere à tipologia do estudo, predominaram as pesquisas de abordagens do tipo descritivas retrospectivas, dos dez estudos analisados, quatro ${ }^{9-12}$ (40\%) mencionam em sua metodologia de pesquisa a abordagem descritiva. Dois estudos ${ }^{13-14}$ (20\%) são relatos de experiência não possuindo amostra de dados. Dois $\operatorname{artigos}^{16,19}$ (20\%) possuem abordagem exploratória, um observacional ${ }^{15}(10 \%)$; um inter-relacional ${ }^{17}$ (10\%). A análise quantitativa é mencionada em apenas um artigo $^{16}$ (10\%), embora sejam apresentados dados coletados, com tratamento estatístico, em seis estudos $^{9-12,15-16}(60 \%)$. 


\section{DISCUSSÃO}

O referencial teórico, utilizado na maioria dos artigos foi o modelo de processo de enfermagem proposto por Wanda de Aguiar Horta. Encontram-se citações diretas à obra da autora e os conceitos de cada fase do processo de enfermagem. Cinco artigos $9,13,15-17$ (50\%) retratam explicitamente realidades institucionais onde 0 referencial teórico utilizado na SAE está fundamentado no modelo de Horta, um deles inclusive traz a referência ao modelo conceitual da autora no título. ${ }^{15}$

A evolução de enfermagem encontra-se associada ao processo de enfermagem e a SAE, sendo descrita como uma de suas fases. Todos os autores enfatizam a sua importância enquanto método instrumental conceitual e técnico necessário para o exercício da enfermagem de forma científica e legal.

A SAE, para $40 \%$ da amostra, necessita de reorganização, ajustes e ações educativas tendo sido apontado dificuldades e inconsistências na implementação nos cenários estudados.

Nesse sentido, destaca-se a correção do excesso de atribuições das enfermeiras, a falta de recursos materiais para o cuidado, insuficiência de pessoal, mesmo quando atendido o quantitativo recomendado de dimensionamento, excesso de tarefas, falta de preparo para utilização desse método de trabalho, resistência na utilização e não valorização do método pelos próprios enfermeiros e demais membros da equipe de enfermagem. ${ }^{9-}$ $10,13,16$
Identificou-se a importância da SAE enquanto método instrumental conceitual e técnico necessário para o exercício da enfermagem de forma científica. Contudo constatam-se deficiências no cumprimento de algumas fases e na documentação das etapas realizadas.

Os artigos analisados constataram falhas na identificação do profissional que realizou registro, bem como a utilização de terminologia incorreta e abreviaturas não convencionais, ilegibilidade, erros de ortografia, emprego de nomes comerciais de artefatos. Percebeu-se pouca expressividade ou anotações e evoluções de enfermagem indistintas quanto ao conteúdo, ausência dos enunciados de diagnósticos de enfermagem, das necessidades psicossociais dos clientes e da existência de um sistema de classificação padronizado na instituição. 11-12,16

Alguns estudos sugerem a padronização dos registros (anotações e evoluções) de enfermagem, reformulação de impressos próprios das fases da SAE nas unidades e elaboração de protocolos assistenciais para a qualificação do registro ${ }^{10,12}$, a revisão evidenciou que existem falhas na implementação da SAE, a qual ocorre de forma fragmentada, tendo com maior frequência apenas 0 registro das etapas de prescrição e histórico realizadas. ${ }^{16}$

Observou-se que mesmo em instituições que já tinham implantado a SAE, a mais de cinco anos, e em instituições destinadas ao ensino e a pesquisa, ainda necessitam de orientação e treinamentos para a 


\section{JOURNALF \\ NURSING \\ AND $\square$ 든}

execução da evolução de enfermagem. ${ }^{12,16}$ Esse fato justifica o investimento em educação permanente, apontado como estratégia para a resolução dos problemas e qualificação da assistência prestada, corroborado com dado encontrado em estudo realizado em uma instituição de ensino que identificou que a maioria, dos enfermeiros investigados, desconhecem a legislação, mesmo os que reconhecem a importância da realização das anotações e evolução de enfermagem. ${ }^{20}$

Entre todas as etapas da SAE identificou-se que a mais negligenciada foi a evolução de enfermagem, evidenciando que 0 processo de enfermagem continua inconsistente em muitos dos ambientes em que se dá o cuidado no Brasil, pois não foram encontrados registros que descrevam o resultado dos cuidados prestados em relação ao diagnóstico e a prescrição de enfermagem. Entendese que a evolução de enfermagem vem contemplando estritamente aspectos de interesse médico em detrimento do seu campo próprio de atuação, descritas sem análise clínica e sem reportar-se devidamente ao cuidado prescrito. ${ }^{9}$

No entanto a qualificação desse processo depende da valorização profissional, visto que a evolução se constitui a etapa da SAE que mais dispenda tempo na sua execuação ${ }^{15}$, bem como da compreensão dos enfermeiros em reconhecer que a SAE, pode facilitar seu trabalho e direcionar a assistência prestada ao cliente. ${ }^{16-17}$

Em quadro estudos 9 ,12,16-17 (40\%) a auditoria de enfermagem foi utilizada
ISSN 2236 - 1987

como método de coleta de dados, tendo sido apontada como instrumento fundamental na avaliação e detecção dos problemas relativos aos registros de enfermagem, pois possibilita a avaliação das ações efetuadas, a orientação para a equipe e a instituição, quanto ao registro apropriado das ações profissionais e o respaldo ético e legal, frente aos conselhos, às associações de classe e a justiça. ${ }^{12,16}$

A existência de um grande volume de inconformidades na implementação e execução dessa sistemática abre um oportuno espaço de atuação para a auditoria de enfermagem a qual poderia contribuir significativamente para o exercício da enfermagem de forma científica, segura, legal e de qualidade.

\section{CONSIDERAÇÕES FINAIS}

A evolução de enfermagem integra o processo de enfermagem e constitui uma das fases da SAE, a qual apresenta inconformidades e deficiências na sua implementação. Fato que evidencia a necessidade de maior investimento e pesquisa acerca do tema, principalmente se considerar-se os avanços tecnológicos ocorridos no setor saúde na atualidade.

A SAE, embora seja um método de consenso, amplamente conhecido e legalmente instituído, ainda enfrenta uma série de inconsistências e problemas em sua implementação e execução. A evolução de enfermagem, enquanto integrante desse sistema, sofre os mesmos efeitos.

Nesse contexto, destaca-se a necessidade de ações de treinamento e 


\section{ISSN 2236 - 1987}

educação permanente, a construção de formulários padronizados e a condescendência dos profissionais em relação à utilidade, à validade e à correta execução das fases do processo de enfermagem. Salienta-se a ênfase na abordagem desse tema desde a graduação, visando à construção de uma conduta prática cotidiana na assistência de enfermagem.

Os possíveis caminhos para contornar esse quadro são: reorganização e padronização da SAE, investimentos em treinamento e educação permanente dos enfermeiros e demais membros da equipe de enfermagem, e atividades de auditoria. Estas seriam estratégias relevantes para promover as mudanças necessárias nos cenários assistenciais.

0 presente estudo, também, evidenciou a existência de algumas lacunas acerca dos motivos da resistência à implementação da evolução de enfermagem, nos hospitais do país, as quais merecem maiores investigações.

\section{REFERENCIAS}

1. Horta WA. Processo de enfermagem. $2^{\mathrm{a}}$ Ed. Rio de Janeiro: Editora Guanabara; 2011.

2. Ministério da Saúde (BR). Secretaria de Atenção à Saúde. Carta dos Direitos dos Usuários de Saúde. Brasília; 2011.

3. Conselho Federal de Enfermagem (BR). Resolução Cofen 514, de 05 de maio de 2016. Aprova o Guia de Recomendações para os registros de enfermagem no prontuário do paciente [Internet]. 2016 [acesso 2016 jul 04]. Disponível em:
content/uploads/2016/08/Guia-deRecomenda\%C3\%A7\%C3\%B5es-CTLNVers\%C3\%A3o-Web.pdf

4. Oliveira RS, Almeida EC, Azevedo NM, Almeida MAP, Oliveira JGC. Reflexões sobre as bases científicas e fundamentação legal para aplicação da sistematização do cuidado de enfermagem. Rev UNIABEU. 2015 set/dez;8 (20):350-62.

5. Garcia TR, Nóbrega MML, Carvalho EC. Nursing process: application to the professional practice. Online braz j nurs. 2004;3(2):25-33.

6. Malucelli A, Otemaier KR, Bonnet $M$, Cubas MR, Garcia TR. Sistema de informação para apoio à Sistema de informação para apoio à Sistematização da Assistência de Enfermagem. Rev bras enferm. 2010 jul-ago;63(4): 629-36.

7.Galvão CM, Sawada NO, Trevizan MA. Revisão sistemática: recurso que proporciona a incorporação das evidências na prática da enfermagem. Rev latino-am enfermagem. 2004 maio/jun;12(3):540-56.

8. Galvão TF, Pansani TSA. Principais itens para relatar revisões sistemáticas e meta-análises: a recomendação PRISMA. Epidemiol serv saúde. 2015 abr-jun;24(2):335-42.

9. Franco MTG, Akemi EM, D'Inocento M. Avaliação dos registros de enfermeiros em prontuários de pacientes internados em unidade de clínica médica. Acta paul enferm [Internet]. 2012 [acesso em 2016 jul 3];25(2):163-70. Disponível em: http://www.scielo.br/pdf/ape/v25n2 /a02v25n2.pdf http://www.cofen.gov.br/wp- 


\section{ISSN 2236 - 1987}

10. Reppetto MÂ, Souza MF. Avaliação da realização e do registro da Sistematização da Assistência de Enfermagem (SAE) em um hospital universitário. Rev bras enferm [Internet]. 2005[acesso em 2016 jul 2];58(3):325-9. Disponível em: http://www.scielo.br/pdf/reben/v58 n3/a14v58n3.pdf

11. Gomes DC, Cubas MR, Pleis LE, Shmeil MAH, Peluci APVD. Termos utilizados por enfermeiros em registros de evolução do paciente. Rev bras enferm. 2016;37 Suppl:S539-27.

12. Setz VG, D'Innocenzo M. Avaliação da qualidade dos registros de enfermagem no prontuário por meio da auditoria. Acta paul enferm [Internet]. 2009[acesso em 2016 jul 2];2(3):313-7. Disponível em: http://www.scielo.br/pdf/ape/v22n3 /a12v22n3.pdf

13. Neves RS. Sistematização da assistência de enfermagem em unidade de reabilitação segundo o modelo conceitual de horta. Rev bras enferm [Internet]. 2006[acesso em 2016 jul 3];59(4):556-9. Disponível em: http://www.scielo.br/pdf/reben/v59 n4/a16v59n4.pdf

14. Vaz AF, Macedo DD, Montagnoli ETL, Lopes MHBM, Grion RC. Implementação do processo de enfermagem em uma unidade de radioterapia: elaboração de instrumento para registro. Rev latinoam enfermagem [Internet]. 2002[acesso em 2016 jul 3];10(3):288$97 . \quad$ Disponível em: http://www.scielo.br/pdf/rlae/v10n3 /13339.pdf

15. Almeida MA, Severo IM, Chaves EB, Barreto LNM, Borba DM. Tempo despendido na execução do processo de enfermagem em um centro de tratamento intensivo. Esc Anna Nery [Internet]. 2012[acesso em 2016 jul 3];16(2):292-6. Disponível em: http://www.scielo.br/pdf/ean/v16n2 112.pdf

16. Neves RS, Shimizu HE. Análise da implementação da Sistematização da Assistência de Enfermagem em uma unidade de reabilitação. Rev bras enferm [Internet]. 2010[acesso em 2016 jul 3];63(2):222-9. Disponível em: http://www.scielo.br/pdf/reben/v63 n2/09.pdf

17. Venturini DA, Marcon SS. Anotações de enfermagem em uma unidade cirúrgica de um hospital escola. Rev bras enferm [Internet]. 2008[acesso em 2016 jul 3];61(5):570-7. Disponível em:

http://www.scielo.br/pdf/reben/v61 n5/a07v61n5.pdf

18. Salgado PO, Tannure MC, Oliveira CR, Chianca TCM. Identificação e mapeamento das ações de enfermagem prescritas para pacientes internados em uma UTI de adultos. Rev bras enferm [Internet]. 2012 mar/abr[acesso em 2016 jul 3];65(2):291-6. Disponível em: http://www.scielo.br/pdf/reben/v65 n2/v65n2a14.pdf

19. Barbosa SF, Tronchin DMR. Manual de monitoreo de la calidad de los registros de enfermería en la atención domiciliaria. Rev bras enferm [Internet]. $2015 \mathrm{mar} / \mathrm{abr}$ [acesso em 2016 jul 3]; 68(2):253-60. Disponível em:

http:/ / www.scielo.br/scielo.php?scrip $\mathrm{t}=\mathrm{sci}$ arttext\&pid=S0034-

$71672015000200253 \&$ lang=pt 


\section{NURSING AND}

20. Borges FFD, Azevedo CT, Amorim TV, Figueiredo MAG, Ribeiro RGM. Importância das anotações de enfermagem segundo a equipe de enfermagem: implicações profissionais e institucionais. Rev enferm cent-oeste min [Internet]. 2017 [acesso em 2017 jul 23];7(1):1-8. Disponível em: http://www.seer.ufsj.edu.br/index.p $\mathrm{hp} / \mathrm{recom} /$ article/view/1147/1310

Data de submissão: 28/02/2017

Data de aceite: $21 / 10 / 2017$

Data de publicação: 26/12/2017 